Cumhuriyet International Journal of Education-CIJE

e-ISSN: $2147-1606$

Vol 6 (1), 2017, $66-81$

\title{
Multiple Representations and Teaching Mathematics: An Analysis of the Mathematics Textbooks
}

\section{Semahat İNCÍKABI}

\begin{abstract}
Summary
The economic, political and educational problems encountered in mathematics education have been influential in defining different approaches and learning theories that can be applied in learning environments. One of these theories is the multiple representation theory of learning. Multiple representations mean that a concept is repeatedly represented by different types of representations, such as verbal, graphical, mathematical representations, and that students are exposed to the same concept several times (Prain \& Waldrip, 2006, p.1844).

According to Even (1998), the skills that are at the focal point of conceptual learning in mathematics are the ability to identify and express the same concept in different forms of representation, to choose the most appropriate representation from among the various representations, and to be aware of the advantages and disadvantages of the representations.

Integrating multiple representations effectively in mathematics teaching provides opportunity to conceptualize, express and observe mathematical concepts in different ways. This allows students to have deeper and more flexible notions about concepts (Hiebert \& Carpenter, 1992; Piez \& Voxman, 1997). In the case of a transition between multiple representations, it can be said that the mathematics cannot be understood at the conceptual dimension (Ainsworth, 1999, Van der Meij \& De Jong, 2006). In this context, teachers are expected to encourage students to use multiple representations in mathematics curricula (NCTM, 2000).

In this study, representation types placed in the secondary school mathematics textbooks were determined and the transitions between these representations were analyzed in the context of in-class and out-of-class activities. Being qualitative in nature, this study utilized document analysis method to analyze the transitions between representations in secondary school mathematics textbooks. In this study, mathematics textbooks were analyzed by considering verbal, algebraic, model, table, graphical and real life representations.

According to research findings, while textbooks contain algebraic representations most, they have significant distributions in verbal and model representations. On the other hand, it is noteworthy that the table, graphic and real life representations are included in the textbooks in a very small proportion. Looking at the transitions between representations, it is seen that the relationship between the representations in the class activities is in significant proportions between algebraic, verbal and model representations. Similarly, in out-of-class activities, the relationship between the representations appears to be in significant proportions between algebraic, verbal and model representations. In addition, secondary school mathematics textbooks prefer tables, real life and graphic representations in the solution of the questions both in- and out-of-class activities.
\end{abstract}

